\title{
A New Fluctuating Asymmetry Index, or the Solution for the Scaling Effect?
}

\section{Cino Pertoldi ${ }^{1,2, *}$ and Torsten Nygaard Kristensen ${ }^{1}$}

1 Department of Chemistry and Bioscience, Aalborg University, Fredrik Bajers Vej 7H, 9220 Aalborg, Denmark; E-Mail: tnk@bio.aau.dk

2 Aalborg Zoo, Mølleparkvej 63, DK-9000 Aalborg, Denmark

* Author to whom correspondence should be addressed; E-Mail: cp@bio.aau.dk; Tel.: +45-9940-3604.

Academic Editor: John H. Graham

Received: 26 January 2015 / Accepted: 23 March 2015 / Published: 1 April 2015

\begin{abstract}
Two principal methods are commonly employed for the estimation of developmental instability at the population level. Some studies use variances of morphological traits $\left(\sigma^{2}\right)$, while others use fluctuating asymmetry (FA). In both cases, differences in the degree of developmental instability can be tested with an $F$-test, which is the most common way to compare variances. However, the variance is expected to scale proportionally to the square of the mean as there is a tendency in biological data for $\sigma_{p}^{2}$ to scale proportionally to the square of the mean $(\bar{\mu}): \sigma_{p}^{2}=Z \bar{\mu} \xi$, where $\xi$ is the scaling exponent, which is expected to be two for pure statistical reasons, $\bar{\mu}$ is the mean of the trait and $Z$ is a measure of individual-level variability. Because of this scaling effect, the fluctuating asymmetry will be affected, FA is estimated as the variance between the right and the left sides of a trait $\left(\sigma^{2}{ }_{r-l}=\sigma^{2}{ }_{r}+\sigma^{2}{ }_{l}-2 r \sigma_{r} \sigma l\right)$, where $\sigma_{r}^{2}$ and $\sigma^{2}$ are the variances of the right and the left trait values, respectively. In this paper, we propose a novel method that allows an exact correction of the scaling effect, which will enable a proper comparison of the degree of fluctuating asymmetry for a trait. The problem of the scaling of the FA with the trait size is quite crucial if FA is to be considered an indicator of fitness or an indicator of environmental or genetic stress, as different stresses or fitness levels are typically accompanied by a change of the traits' $\bar{\mu}$.
\end{abstract}

Keywords: Taylor's power law; developmental instability; $F$-test 


\section{Introduction}

\subsection{Developmental Instability}

The development of a trait in a given environment is disturbed by random processes that cause it to deviate from its expected phenotype. It is believed that an individual's ability to buffer its development against these random perturbations is influenced by genotype, environment and/or genotype-environment interactions [1-4]. Developmental instability (DI) results when stress affects the buffering capacity of the processes that provide stability to an organism's development [5]. The theoretical argument that stressed individuals have greater DI is supported by some research showing a positive relationship between DI and the intensity of stress [4,6-12]. However, empirical studies supporting its general adequacy for monitoring species or populations are generally lacking or contradictory [4,13-16].

Two principal methods are commonly employed for the estimation of DI at the population level. Some studies use variances of morphological characters or phenotypic variance $\left(\sigma^{2}\right)$ [12,16-18], where the estimate may be blurred by genetic variation $\left(\sigma^{2}\right)$, while other studies use fluctuating asymmetry (FA) $[1,4,7]$.

Phenotypic variance can be considered as an estimator of DI when variation caused by environmental variability $\left(\sigma^{2}\right.$ e and $\sigma_{\mathrm{g}}^{2}$ is negligible [19]. Several studies have revealed that the $\sigma_{\mathrm{p}}^{2}$ of quantitative traits increases in populations experiencing environmental stress $[12,16,20]$. The problem with estimating $\sigma^{2} p$ even in a monoclonal strain $\left(\sigma^{2} g=0\right)$ is that the estimate in general will be strongly affected by $\sigma^{2}[17,19,21,22]$.

\subsection{Phenotypic Variance}

In biological data there is a tendency for $\sigma_{\mathrm{p}}^{2}$ to scale proportionally to the square of the mean $(\bar{\mu})[23]$ :

$$
\sigma_{\mathrm{p}}^{2}=Z \bar{\mu}^{\xi}
$$

where $\xi$ is the scaling exponent, which is expected to be two for pure statistical reasons (see [23] for derivation). $Z$ is a measure of individual-level variability [23]. The regression of $\log \sigma^{2}$ (dependent) on $\log \bar{\mu}$ (independent) gives a line with a slope of two, called Taylor's power law [24,25]. However, $\xi$ has been found to be considerably smaller than two for many morphological traits $(\xi<2)[26]$.

\subsection{Fluctuating Asymmetry}

Whereas it is generally accepted that $\sigma_{\mathrm{p}}^{2}$ is influenced by $\sigma_{\mathrm{g}}^{2}$ and $\sigma_{\mathrm{e}}^{2}$ [27], FA at the individual level has the advantage that dissimilarity in the expression of a given character on the left and right sides of an organism cannot be explained by either differences in genotype or environment $[1,7]$.

Following Palmer and Strobeck [1], FA can be estimated as FA $=\sigma^{2} r-l$, where $r$ and $l$ are, respectively, the trait values on the right and left side. A scaled FA index can be utilized by dividing FA with the mean of the traits: $\left(\sigma^{2} r-l\right) /(0.5 r+0.5 l)($ FA7 index; [1]). Reference [1] suggested also the index FA3 $=\bar{\mu}_{I r-l I} /(0.5 r+0.5 l)$, which is equivalent to the mean of the absolute value of the difference between the left and right side divided by the population trait size; however, the unsigned difference between the $r$ and the $l$ side is not normally distributed, and therefore, other statistical problems are introduced by using this index, which require testing with non-parametric techniques [1]. The division 
by the mean trait size can correct for scale differences. This is a necessary operation when the $\left(\sigma^{2} r-l\right)$ is positively correlated with the mean of the trait. Other indices (FA9 and FA9a; [1]) that are equivalent to one minus the square of the correlation coefficient between the right and left sides $\left(1-r_{r, l}^{2}\right)$ (FA9; [1]) or one minus the correlation coefficient between the right and left $\left(1-r_{r, l}\right)$ (FA9a; [1]) have also been proposed [1,28]. The performance of the different indices' depends on whether the FA of a trait is more correlated to its $\bar{\mu}$ or its $\sigma_{\mathrm{p}}^{2}[28]$.

Windig and Nylin [28] have shown that FA measured as $\left(\sigma^{2} r-l\right)$ on 36 traits in the Speckled Wood Butterfly (Pararge aegeria) were highly correlated both with the mean $(r=0.94)$ and the variance $(r=0.96)$. As a consequence of this strong correlation between $\left(\sigma^{2}{ }_{r-l}\right)$ with the $\sigma_{\mathrm{p}}^{2}$ and $\bar{\mu}$ of the trait, the same problems mentioned above for the standardization of the variance with the mean will exist for the standardization of the $\left(\sigma^{2} r-l\right)$ with the mean. In order to get an accurate estimate of the level of FA for a given trait new as the different indices suggested by $[1,28]$ and have to be considered as different options for correcting the scaling effect are needed. This will allow a comparison of the level of FA estimated for traits with different means or for the same trait, but with a different means in different samples. Correcting for scale effects can however, increase the possibility of committing errors of Type 1 and Type 2. Considering the fact that often, the differences of the FA levels are subtle, makes the need for a methodology that can correct for the scaling effect in a precise way that do need effect the likelihood to make Type 1 and Type 2 errors quite urgent. The aim of this paper is therefore to propose a new methodology that will allow an exact correction for the scaling effect.

\section{Methods and Results}

In this paper, we suggest the following method:

A. We assume two sample sizes: $\left(\bar{\mu}_{1},\left(\sigma^{2} r-l\right) 1\right.$ and $\left.\bar{\mu}_{2},\left(\sigma^{2} r-l\right)_{2}\right)$ in which the $\bar{\mu}$ and the $\left(\sigma^{2} r-l\right)$ will be log-transformed. For both samples, also the correlation between the right and the left values will be estimated, and we will therefore have two correlations: $r_{1}$ and $r_{2}$.

B. The $\left(\sigma^{2} r-l\right)$ is equivalent to:

$$
\left(\sigma^{2} r-l\right)=\sigma_{r}^{2}+\sigma^{2}-2 r \sigma_{r} \sigma l
$$

as $\sigma^{2}{ }_{r}=\sigma^{2}{ }_{l}=\sigma^{2}$.

We substitute (from Equation (1)):

$$
\left(\sigma^{2}{ }_{r-l}\right)=Z \bar{\mu}_{1}{ }^{\xi}+Z \bar{\mu}_{2}{ }^{\xi}-2 \mathrm{r}\left(Z \bar{\mu}_{1}^{\xi} Z \bar{\mu}_{2}\right)^{1 / 2}
$$

Rearranging:

$$
\left(\sigma^{2} r-l\right)=2 Z \bar{\mu}^{\xi}-2 r Z \bar{\mu}^{\xi}
$$

and:

$$
\left(\sigma^{2} r-l\right)=2 Z \bar{\mu} \xi(1-r)
$$

C. Following a log-transformation, Equation (3) becomes:

$$
\log \left(\sigma^{2} r-l\right)=\log 2+\log Z+\log (1-r)+\xi \cdot \log \bar{\mu}
$$

Consequently, the regression of $\log \left(\sigma^{2} r-l\right)$ (dependent) on $\log \bar{\mu}$ (independent) gives a line with an intercept equal to: $(\log 2+\log Z+\log (1-r))$ and a slope of $\xi=2$ for pure statistical reasons. In this 
equation, it is assumed that $r_{1}=r_{2}$, which means that there is no difference in the degree of correlation between the right and the left values measured in the two samples with two means: $\bar{\mu}_{1}$ and $\bar{\mu}_{2}$; however $\left(\sigma^{2} r-l\right)_{1} \neq\left(\sigma_{r-l}^{2}\right)_{2}$, because, despite the fact that $r_{1}=r_{2}$, the $\log \left(\sigma^{2} r-l\right)$ is increasing with a slope of two $(\xi=2)$. This $\xi$ is therefore a hypothetical slope, which will only coincide with the observed slope if $r_{1}=r_{2}$, and we will therefore call it $\xi\left(\xi_{i}\right)=2$.

D. Estimation of the slope of the line using the observed values of: $\log \bar{\mu}_{1}, \log \left(\sigma^{2} r-l\right)_{1}$ and $\log \bar{\mu}_{2}, \log \left(\sigma^{2} r-l\right) 2$; therefore, we will obtain an observed $\xi\left(\xi_{o}\right)$, which will be equivalent to: $\xi_{o}=\left[\log \left(\sigma_{r}^{2}-l\right)_{2}-\log \left(\sigma_{r-l}^{2}\right)_{1}\right] /\left(\log \bar{\mu}_{2}-\log \bar{\mu}_{1}\right)$.

E. Subtraction of the observed slope $\left(\xi_{o}\right)$ from the hypothetical slope $\left(\xi_{i}\right)=2$; therefore Equation (4) becomes:

$$
\log \left(\sigma^{2} r-l\right)=\log 2+\log Z+\log (1-r)+\left(\xi_{o}-\xi_{i}\right) \log \bar{\mu}
$$

which allows an estimation of the corrected values of the log-variance $\log \left(\sigma^{2} r-l\right)_{\mathrm{c}}$ as:

$$
\left(\log \left(\sigma^{2} r-l\right)_{\mathrm{c}}-\log \left(\sigma^{2} r-l\right)_{1}\right) /\left(\log \bar{\mu}_{2}-\log \bar{\mu}_{1}\right)=\left(\xi_{o}-\xi_{i}\right),
$$

Resolving:

$$
\log \left(\sigma^{2} r-l\right)_{\mathrm{c}}=\left(\xi_{o}-\xi_{i}\right)\left(\log \bar{\mu}_{2}-\log \bar{\mu}_{1}\right)+\log \left(\sigma^{2} r-l\right) 1
$$

F. Estimation of the anti-log value of $\log \left(\sigma^{2} r-l\right)$.

G. $F$-test for testing differences between $\left(\sigma^{2} r-l\right) \mathrm{c}$ and $\left(\sigma^{2} r-l\right) 1$.

Therefore, the correction can be easily applied in the form of an FA index, which we will call for commodity the "FA corrected for scaling index $(\mathrm{FA} c)$ ", where $\mathrm{FA}_{\mathrm{cs}}=\log \left(\sigma^{2} r-l\right)_{\mathrm{c}}$ and consequently $\mathrm{FA}_{\mathrm{cs}}=\left(\xi_{o}-\xi_{i}\right)\left(\log \bar{\mu}_{2}-\log \bar{\mu}_{1}\right)+\log \left(\sigma^{2} r-l\right) 1$. Substituting $\left(\xi_{i}\right)=2$ and $\xi_{o}=\left[\log \left(\sigma^{2} r-l\right)_{2}-\log \left(\sigma^{2} r-l\right){ }_{1}\right]$ $\left(\log \bar{\mu}_{2}-\log \bar{\mu}_{1}\right)$, we obtain:

$$
\mathrm{FA}_{\mathrm{cs}}=\left\{\left[\log \left(\sigma^{2} r-l\right) 2-\log \left(\sigma^{2} r-l\right)_{1}\right] /\left[\left(\log \bar{\mu}_{2}-\log \bar{\mu}_{1}\right)\right]-2\right\}\left(\log \bar{\mu}_{2}-\log \bar{\mu}_{1}\right)+\log \left(\sigma^{2} r-l\right)_{1},
$$

in the equation above, there are only four terms: $\log \left(\sigma^{2}{ }_{r-l}\right) 2, \log \left(\sigma^{2}{ }_{r-l}\right) 1, \log \bar{\mu}_{2}$ and $\log \bar{\mu}_{1}$, which are all known and that can easily be estimated in order to obtain the FAcs index, which, after an antilog transformation, will be compared to $\left(\sigma^{2} r-l\right) 1$, with an $F$-test.

\section{Discussion}

\subsection{Scaling Problems of Fluctuating Asymmetry}

Despite the fact that the finding of an exact way to correct FA for scaling could give an impression of a rather technical discussion, the problem of the scaling of the FA with the trait size is crucial if FA has to be considered an indicator of fitness, as often, variation of fitness among individuals is accompanied by a variation of the $\bar{\mu}$ of the traits [7,29,30]. For FA to be considered an indicator of environmental or genetic stress, the problem of scaling is also present as a higher stress level is typically accompanied by a reduction of the traits' $\bar{\mu}[3,16]$. 


\subsection{Transformations}

The log-transformation of the variance [31], the Box-Cox power transformation [32], or the coefficient of variation (CV) [33] are commonly used to make $\sigma^{2}$ independent of $\bar{\mu}$ [34]. The transformed data can be compared with the log-log-test [31], the naive test [34], the likelihood ratio test, Bennett's test, the score test, Miller's test, Doornbos and Dijkstra's test or the Wald test.

All of these tests are used for taking into account eventual violations of the assumptions required by the $F$-test (like for example a small sample size or non-normally distributed data) [23].

\subsection{Correction for the Scaling Effect}

Pertoldi et al. [23] proposed an exact methodology that can correct for the scaling effect of the $\sigma_{\mathrm{p}}^{2}$ with the $\bar{\mu}$ without the need for transformations, which will give an approximate estimate of the true $\sigma^{2}$.

It should also be noted that in Equation (5), $\xi_{o}>\xi_{i}$, the slope of the corrected line $\left(\xi_{o}-\xi_{i}\right)$ continues to remain positive (slope $>0$ ), even if the slope is reduced, which means that $\log \left(\sigma^{2}{ }_{r}-l\right)_{\mathrm{c}}>\log \left(\sigma^{2} r-l\right)_{1}$, which implies that the uncorrected $F$-test is more prone to an error of Type 1 (incorrect rejection of the $\mathrm{H}_{0}$ hypothesis) compared to the $F$-test, which is performed on the corrected variances. If, in Equation (5), $\xi_{o}<\xi_{i}$, the slope of the corrected line will have a negative slope (slope $<0$ ). A negative slope implies that $\left(\sigma_{r-l}^{2}\right)_{\mathrm{c}}<\left(\sigma_{r-l}^{2}\right)$, which is in contradiction to the one-tailed $\mathrm{H}_{\mathrm{A}}$ hypothesis of the $F$-test in which $\sigma_{1}^{2}>\sigma_{2}^{2}$. In fact, $F$-tests that have a $\xi_{o}>\xi_{i}$ may lead to wrong conclusions as the $\sigma^{2}$ in the nominator of the $F$-test (which is supposed to be bigger) is in reality smaller than the $\sigma^{2}$ in the denominator (which is supposed to be smaller). If $\xi_{o}=\xi_{i}$, then the corrected line will have a slope equal to zero.

\subsection{How Can the Scaling Effect Have Afflicted Investigations?}

The possible scenarios listed above are underlying potential problems, which could have afflicted several investigations in many scientific disciplines that have formulated hypotheses based on the $F$-distribution. The bigger the difference between the two means $\left(\bar{\mu}_{1}\right.$ and $\left.\bar{\mu}_{2}\right)$ of the distributions compared in the $F$-test, the higher is the probability that the above-mentioned errors could have led to wrong conclusions. The transformations and approximations listed in this paper can in some way reduce the probability that these errors occur, but at the same time, they can also introduce a further bias and complexity due to the interactions between the scaling effect and the effects on the data transformations.

\section{Conclusions}

The method proposed in this paper can be utilized to make an exact correction for differences in means between samples that are compared. This can be utilized in future investigations dealing with developmental instability.

However even with this correction we should be aware of confounding factors, such as the standard error of the variance and the presence of mixtures due to environmental variability, which can produce platykurtic or leptokurtic distributions of the $(r-l)$ values [35-40]. Mixtures due to genetic substructuring can also produce the same effects that environmental variability can have on the distribution of $(r-l)$ values [41]. The measurement error should be minimal compared to the level of variation of the $(r-l)$ values $[1,42]$ and there should not be antisymmetry and/or directional asymmetry, which will make the 
correction proposed in this paper inapplicable $[43,44]$. Potentially, there is a very promising application of fluctuating asymmetry as an indicator of environmental stress if we choose to apply the exact correction proposed in this paper on clonal organisms where no genetic substructure is present [45-51]. The absence of genetic substructure is allowing us to exclude one of the potential biases which is afflicting the fluctuating asymmetry indexes. In addition, following the methodology proposed in $[21,37,40]$, it will be possible to exclude samples in an investigation where the presence of environmental variability and/or antisymmetry/directional asymmetry have been detected. Once all these checks have been performed on the samples which are being compared for differences in the degree of fluctuating asymmetry, we believe that fluctuating asymmetry remains a powerful tool for comparing the level of developmental instability of samples. The methodology proposed in $[37,40]$ can clearly also be utilized for the detection of the presence of genetic substructuring and in this case fluctuating asymmetry can be utilized for the detection of genetic and environmental stress in sexually reproducing populations which expands its application in the field of conservation biology in wild populations.

\section{Acknowledgments}

This study has been partly supported by the Danish Natural Science Research Council (Grant Numbers \#21-01-0526, \#21-03-0125, 95095995, and \#4002-00036) and the Aalborg Zoo Conservation Foundation (AZCF). Finally, we thank Volker Loeschcke, John Graham and two anonymous reviewers for invaluable suggestions and help.

\section{Author Contributions}

Both authors contributed equally.

\section{Conflicts of Interest}

The authors declare no conflict of interest.

\section{References}

1. Palmer, A.R.; Strobeck, C. Fluctuating asymmetry: Measurement, analysis, patterns. Annu. Rev. Ecol. Syst. 1986, 17, 391-421.

2. Lens, L.; van Dongen, S.; Kark, S.; Matthysen, E. Fluctuating asymmetry as an indicator of fitness: Can we bridge the gap between studies? Biol. Rev. 2002, 77, 27-38.

3. Pertoldi, C.; Andersen, D.H.; Kristensen, T.N.; Loeschcke, V. Consequences of a reduction in genetic variance on developmental instability estimators. Evol. Ecol. Res. 2003, 5, 893-902.

4. Graham, J.H.; Raz, S.; Hel-Or, H.; Nevo, E. Fluctuating Asymmetry: Methods, Theory, and Applications. Symmetry 2010, 2, 466-540.

5. Lens, L.; van Dongen, S.; Galbusera, P. Developmental instability and inbreeding natural bird populations exposed to different levels of habitat disturbance. J. Evol. Biol. 2000, 13, 889-896.

6. Leary, R.F.; Allendorf, F.W. Fluctuating asymmetry as an indicator of stress: implication for conservation biology. Trends Ecol. Evol. 1989, 4, 214-217. 
7. Møller, A.P.; Swaddle, J.P. Asymmetry, Developmental Stability and Evolution; Oxford University Press: Oxford, UK, 1997.

8. Pertoldi, C.; Loeschcke, V.; Madsen, A.B.; Randi, E. Developmental stability in the Eurasian Otter (Lutra lutra) in Denmark. Ann. Zool. Fenn. 1997, 34, 187-196.

9. Pertoldi, C.; Madsen, A.B.; Randi, E.; Braun, A.; Loeschcke, V. Variation of skull morphometry of Eurasian otters (Lutra lutra) in Denmark and Germany. Ann. Zool. Fenn. 1998, 35, 87-94.

10. Graham, J.H.; Freeman, D.C.; Emlen, J.M. Developmental stability: A sensitive indicator of populations under stress. In Environmental Toxicology and Risk Assessment; ASTM STP 1179; Landis, W.G., Hughes, J., Lewis, M.A., Eds.; American Society for Testing and Materials: Philadelphia, PA, USA, 1993; pp. 136-158.

11. Markow, T.A. Developmental Instability: Its Origins and Evolutionary Implications; Kluwer: Dordrecht, The Netherlands, 1994.

12. Kristensen, T.N.; Pertoldi, C.; Pedersen, L.D.; Andersen, D.H.; Bach, L.A.; Loeschcke, V. The increase of fluctuating asymmetry in a monoclonal strain of collembolans after chemical exposure-Discussing a new method for estimating the environmental variance. Ecol. Indic. 2004, 4, 73-81.

13. Vøllestad, L.A.; Hindar, K.; Møller, A.P. Meta-analysis of fluctuating asymmetry in relation to heterozygosity. Heredity 1999, 83, 206-218.

14. Woods, R.E. The association between fluctuating asymmetry, trait variability, trait heritability and stress: A multiply-replicated experiment on combined stresses in Drosophila melanogaster. Evolution 1999, 53, 493-505.

15. Gilligan, D.M.; Woodworth, L.M.; Montgomery, M.E.; Nurthern, R.K.; Briscoe, D.A.; Frankham, R. Can fluctuating asymmetry be used to detect inbreeding and loss of genetic diversity in endangered populations? Anim. Conserv. 2000, 3, 97-104.

16. Pertoldi, C.; Kristensen, T.N.; Andersen, D.H.; Loeschcke, V. Review: Developmental Instability as an estimator of genetic stress. Heredity 2006, 96, 122-127.

17. Imasheva, A.G.; Loeschcke, V.; Lazebny, O.; Zhivotovsky, L.A. Effects of extreme temperatures on quantitative variation and developmental stability in Drosophila melanogaster and Drosophila buzzatii. Biol. J. Linn. Soc. 1997, 61, 117-126.

18. Kristensen, T.N.; Pertoldi, C.; Andersen, D.H.; Loeschcke, V. The use of fluctuating asymmetry and phenotypic variability as indicators of developmental instability: A test of a new method employing clonal organisms and high temperature stress. Evol. Ecol. Res. 2003, 5, 53-68.

19. Lajus, D.L.; Graham, J.H.; Kozhara, A.V. Developmental instability and the stochastic component of total phenotypic variance. In Developmental Instability: Causes and Consequences; Polak, M., Ed.; Oxford University Press: Oxford, UK, 2003; pp. 343-363.

20. Loeschcke, V.; Bundgaard, J.; Barker, J.S.F. Variation in body size and life history traits in Drosophila aldrichi and D. buzzatii from a latitudinal cline in eastern Australia. Heredity 2000, 85, 423-433.

21. Pertoldi, C.; Kristensen, T.N.; Loeschcke, V. A new method for estimating environmental variability for parthenogenetic organisms, and the use of fluctuating asymmetry as an indicator of developmental stability. J. Theor. Biol. 2001, 4, 407-410. 
22. Pertoldi, C.; Loeschcke, V.; Scali, V. Developmental stability in sexually reproducing and parthenogenetic populations of Bacillus rossius rossius and Bacillus rossius redtenbacheri. Evol. Ecol. Res. 2001, 4, 449-463.

23. Pertoldi, C.; Bundgaard, J.; Loeschcke, V.; Barker, J.S.F. The phenotypic variance gradient? A novel concept. Ecol. Evol. 2014, 22, 4230-4236.

24. Taylor, L.R. Aggregation, variance and the mean. Nature 1961, 189, 732-735.

25. Mutsunori, T. On the mathematical basis of the variance-mean power relationship. Res. Popul. Ecol. 1995, 37, 43-48.

26. Fisher, R.A. The relation between variability and abundance shown by the measurements of the eggs of British nesting birds. Proc. R. Soc. Lond. B. Biol. 1937, 122, 1-26.

27. Falconer, D.; Mackay, T. Introduction to Quantitative Genetics, 4th ed.; Longman Inc.: New York, NY, USA, 1996; p. 464.

28. Windig, J.J.; Nylin, S. How to compare fluctuating asymmetry of different traits. J. Evol. Biol. 2000, 13, 29-37.

29. Pertoldi, C.; Bach, L.A.; Madsen, A.B.; Randi, E.; Loeschcke, V. Morphological variability and developmental instability in subpopulations of the Eurasian badger (Meles meles) in Denmark. $J$. Biogeogr. 2003, 30, 949-958.

30. Pertoldi, C.; Givskov, J.S.; David J.R.; Loeschcke, V. Lerner's theory on the genetic relationship between heterozygosity, genomic coadaptation and developmental instability. Evol. Ecol. Res. 2006, $8,1487-1498$.

31. Neves, H.H.R.; Carvalheiro, R.; Queiroz, S.A. Genetic and environmental heterogeneity of residual variance of weight traits in Nellore beef cattle. Genet. Sel. Evol. 2012, 44, doi:10.1186/1297-9686-44-19.

32. Ronnegard, L.; Valdar, W. Detecting major genetic loci controlling phenotypic variability in experimental crosses. Genetics 2011, 188, 435-447.

33. Levy, S.F.; Siegal, M.L. Network hubs buffer environmental variation in Saccharomyces cerevisiae. PLoS Biol. 2008, 6, doi:10.1371/journal.pbio.0060264.

34. Geiler-Samerotte, K.A.; Bauer, C.R.; Li, S.; Ziv, N.; Gresham, D.; Siegal, M.L. The details in the distributions: Why and how to study phenotypic variability. Curr. Opin. Biotechnol. 2013, 24, 752-759.

35. Pertoldi, C.; Faurby, S.; Reed, D.H.; Knape, J.; Björklund, M.; Lundberg, P.; Kaitala, V.; Loeschcke, V.; Bach, L.A. Scaling of the mean and variance of population dynamics under fluctuating regimes. Theor. Biosci. 2014, 133, 165-173.

36. Pertoldi, C.; Faurby, S. Consequences of Environmental Fluctuations on Taylor's Power Law and Implications for the Dynamics and Persistence of Populations. Acta Biotheor. 2013, 61, 173-180.

37. Pertoldi, C.; Jorgensen, H.B.H.; Randi, R.; Jensen, L.F.; Kjaersgaard, A.; Loeschcke, V.; Faurby, S. Implementation of mixture analysis on quantitative traits in studies of neutral versus selective divergence. Evol. Ecol. Res. 2012, 14, 881-895.

38. Pertoldi, C.; Bach, L.A.; Loeschcke, V. On the brink between extinction and persistence. Biol. Direct 2008, 3, doi:10.1186/1745-6150-3-47.

39. Pertoldi, C.; Bach, L.A.; Barker, J.S.F.; Lundberg, P.; Loeschcke, V. The consequences of the variance-mean rescaling effect on effective population size. Oikos 2007, 116, 769-774. 
40. Pertoldi, C.; Garcia-Perea, R.; Godoy, J.A.; Delibes, M.; Loeschcke, V. Morphological consequences of range fragmentation and population decline on the endangered Iberian lynx (Lynx pardinus). J. Zool. 2006, 268, 73-86.

41. Pertoldi, C.; Loeschcke, V.; Braun, A.; Madsen, A.B.; Randi, E. Craniometrical variability and developmental stability. Two useful tools for assessing the population viability of Eurasian otter (Lutra lutra) populations in Europe. Biol. J. Linn. Soc. 2000, 70, 309-323.

42. Björklund, M.; Merilä, J. Why some measures of fluctuating asymmetry are so sensitive to measurement error. Ann. Zool. Fenn. 1997, 34, 133-137.

43. Petavy, G.; David, J.R.; Debat, V.; Pertoldi, C.; Moreteau, B. Phenotypic and genetic variability of sternopleural bristle number in Drosophila melanogaster under daily thermal stress: Developmental instability and anti-asymmetry. Evol. Ecol. Res. 2006, 8, 149-167.

44. Pertoldi, C.; Podesta, M.; Loeschcke, V.; Schandorff, S.; Marsili, L.; Mancusi, C.; Nicolosi, P.; Randi, E. Effect of the 1990 die-off in the northern Italian seas on the developmental stability of the striped dolphin Stenella coeruleoalba (Meyen, 1833). Biol. J. Linn. Soc. 2000, 71, 61-70.

45. Andersen, D.H.; Pertoldi, C.; Scali, V.; Loeschcke, V. Intraspecific hybridization, developmental stability and fitness in Drosophila mercatorum. Evol. Ecol. Res. 2002, 4, 603-621.

46. Andersen, D.H.; Pertoldi, C.; Scali, V.; Loeschcke, V. Heat stress and age induced maternal effects on wing size and shape in parthenogenetic Drosophila mercatorum. J. Evol. Biol. 2005, 18, 884-892.

47. Andersen, D.H.; Pertoldi, C.; Loeschcke, V.; Scali, V. Developmental instability, hybridization and heterozygosity in stick insects of the genus Bacillus (Insecta; Phasmatodea) with different modes of reproduction. Biol. J. Linn. Soc. 2006, 87, 249-259.

48. Faurby, S.; Kjaersgaard, A.; Pertoldi, C.; Loeschcke, V. The effect of maternal and grandmaternal age in benign and high temperature environments. Exp. Gerontol. 2005, 40, 988-996.

49. Kjaersgaard, A.; Faurby, S.; Andersen, D.H.; Pertoldi, C.; David, J.R.; Loeschcke, V. Effects of temperature and maternal and grandmaternal age on wing shape in parthenogenetic Drosophila mercatorum. J. Therm. Biol. 2007, 32, 59-65.

50. Rogilds, A.; Andersen, D.H.; Pertoldi, C.; Dimitrov, K.; Loeschcke, V. Maternal and grandmaternal age effects on developmental instability and wing size in parthenogenetic Drosophila mercatorum. Biogerontology 2005, 6, 61-69.

51. Pertoldi, C.; Rogilds, A.; Andersen, D.H.; Loeschcke, V. Heat-induced maternal effects in Drosophila mercatorum and its evolutionary consequences. Evol. Ecol. Res. 2005, 7, 203-217.

(C) 2015 by the authors; licensee MDPI, Basel, Switzerland. This article is an open access article distributed under the terms and conditions of the Creative Commons Attribution license (http://creativecommons.org/licenses/by/4.0/). 